\title{
A SITUAÇÃO FINANCEIRA DOS ESTADOS BRASILEIROS: UMA PROPOSTA UTILIZANDO ANÁLISE DISCRIMINANTE
}

\section{BRAZILIAN STATES FINANCIAL SITUATION VERIFYING: A PROPOSAL WITH THE USAGE OF DISCRIMINANT ANALYSIS}

\author{
GILMAR RIBEIRO DE MELLO \\ Doutorando do Programa em Controladoria e Contabilidade \\ pela Universidade de São Paulo \\ E-mail: gmello@usp.br
}

\author{
VALMOR SLOMSKI \\ Professor Doutor do Departamento de Contabilidade e \\ Atuária da Universidade de São Paulo \\ E-mail: valmor@usp.br
}

\section{Resumo}

Quando se estuda a situação financeira das entidades públicas, depara-se com vários indicadores, com formas ou fórmulas de cálculo diferentes, para as quais cada autor procura demonstrar as justificativas que melhor as representem. Nesse contexto, o objetivo deste trabalho é propor uma função discriminante, em respeito aos indicadores que representam a situação financeira dos estados brasileiros. Para tanto, foram selecionados os indicadores relacionados à parte financeira, calculados sobre o período de 2000 a 2005, em todos os Estados. Em seguida, aplicou-se a técnica estatística "análise discriminante". Considerando os resultados, depois que os pressupostos foram atendidos, concluiu-se que as variáveis que melhor representam a situação financeira dos estados brasileiros são as que fornecem as receitas per capita (RP / População); e a relação entre ativo total menos o passivo total e a receita corrente líquida ((AT - PT) / $\mathrm{RCL}$ ). Dessa forma, demonstra-se que a situação financeira não se explica por um único indicador, é necessário um conjunto de indicadores reunidos estatisticamente.

Palavras-chave: situação financeira; estados brasileiros; análise discriminante.

\section{Abstract}

When Public entities' financial situation is studied, we face many indicators and different ways and formulae to calculate them. Each author seeks to demonstrate these ways on his own manner, on a mean that according to his justification best represents them. In this context, the objective of this paper is to propose a discriminant function with the indicators that represent Brazilian states financial situation. In order to achieve this objective, there had been selected indicators related to finances, calculated for the period of 2000 to 2005, for every Brazilian state. Afterwards, Discriminant Analysis statistics technique was applied. Considering the results, after attending the presuppositions, it was concluded that the variables which best represent Brazilian states financial situation are those providing per capita income (RP/Population) and the relation between total assets (AT) less the total liability (PT) and net current revenue (RCL) [(AT - PT)/ RCL]. Thus, it is demonstrated that the financial situation is not explained by a single indicator, and it is necessary a set of indicators statistically reunited.

Key words: financial situation, Brazilian states, discriminant analysis. 


\section{INTRODUÇÃO}

A constituição de 1988 aumentou a descentralização fiscal no Brasil, proporcionando aos Estados ganhos de receitas com 0 acréscimo dos fundos de participação, mas por outro lado, esses Estados não tiveram a obrigação constitucional de elevar, proporcionalmente, suas responsabilidades. Tal descentralização de receitas e indisciplina fiscal tornou mais evidente a insuficiência dos controles institucionais sobre as finanças públicas estaduais; o que resultou em uma crise fiscal na maioria dos estados brasileiros, aumentando a freqüência do socorro financeiro por parte do Governo Federal.

A situação financeira dos Estados é resultado de determinações que refletem o quadro geral da evolução do setor público brasileiro e não apenas a condição particular de determinada unidade. A trajetória das finanças estaduais tem de ser pensada nos marcos, de um lado, da crise fiscal dos anos 80 , da política de estabilização e da renegociação da dívida pública e; de outro, do baixo crescimento da economia. Essas condições provocaram um aumento das obrigações financeiras dos Estados e não geraram, simultaneamente, capacidade de pagamento dos encargos, obstruindo o caminho de saída da crise.

A evolução financeira estadual dependia das decisões federais sobre o montante da dívida a ser rolada e sobre o acesso a novos créditos. A fixação desses parâmetros definia o resultado das contas públicas. Além disso, cresceu o peso da articulação financeira com as empresas e com os próprios bancos instrumentos de financiamento estadual. Assim, o poder de gasto dependia das condições favoráveis de rolagem da dívida e do acesso a novos créditos. As conseqüências foram a situação de crise latente e a crescente deterioração das finanças estaduais.

Para Cardoso (2003), a situação financeira estadual ou municipal é dramática, uma vez que grande parte dos seus recursos é para pagar as despesas com pessoal ou para pagar as dívidas com a própria união.

Além da situação financeira exposta acima, os gestores das entidades públicas e a sociedade (stakeholders), têm a sua disposição inúmeros indicadores (com várias formas ou fórmulas de cálculo, propostas por vários autores) ligados à situação financeira, que muitas vezes não explicam a verdadeira disponibilidade existente.

Analisando essas diferentes formas, percebe-se uma pluralidade de métodos pelos quais cada autor procura demonstrar, de maneira diferente, a partir de justificativas próprias, as situações financeiras.

Assim sendo, o problema de pesquisa deste artigo é: quais indicadores, cujos dados são extraídos das demonstrações contábeis, podem representar a situação financeira dos estados brasileiros?

Nesse contexto, o objetivo é propor uma função discriminante com os indicadores que melhor representem a situação financeira desses Estados.

\section{REVISÃO BIBLIOGRÁFICA}

Manter uma sólida situação financeira é um dos pilares da operação eficiente e eficaz do governo. A situação financeira de um governo tende a ser invisível ao público e a muitos gestores públicos, até que surge uma emergência financeira. Quando a emergência financeira se torna visível, os problemas financeiros de um governo tornam-se, freqüentemente, tão severos que medidas draconianas são requisitadas para manter a 
solvência financeira, como por exemplo: demissão dos principais funcionários, cortes no orçamento, aumento nos impostos etc. (DUNCOMBE et al, 2003, p. 1).

De acordo com o Office of State Comptroller (2002, p. 1), os gestores locais devem periodicamente avaliar a situação financeira de seu governo, pois essa análise pode provêlos de informações valiosas do passado, presente e futuro sobre as finanças da entidade. Dessa forma, eles poderão fazer ações para minimizar as fraquezas e fortalecer a saúde fiscal, podendo também, assegurar melhorias nos recursos que estão disponíveis para financiar o nível e qualidade dos serviços esperados pelo cidadão.

Para Cica (1997, p. 5-6), a situação financeira de um governo é saúde financeira medida por sustentabilidade, vulnerabilidade e flexibilidade, no contexto do ambiente econômico e financeiro global. Sendo que: sustentabilidade diz respeito a até que ponto um governo pode manter os programas existentes e satisfazer os requerimentos dos credores sem aumentar a carga da dívida na economia; vulnerabilidade diz respeito a até que ponto um governo pode aumentar seus recursos financeiros para responder ao surgimento de compromissos, pela expansão de suas receitas ou aumento da carga da dívida; e flexibilidade diz respeito a até que ponto um governo torna-se dependente, portanto vulnerável, pela influência ou controle de fontes de financiamentos externos ou internos.

Com relação aos aspectos financeiros, Matias e Campello (2000, p. 237) nos mostram que eles se referem às necessidades e à utilização dos recursos financeiros, relacionando a natureza e estrutura da captação com a natureza e estrutura de aplicação; e quanto à análise dos aspectos, vários fatores devem ser equacionados, dos quais se destacam: endividamento, estrutura de capital, ativos, liquidez, estrutura de receitas, estrutura de despesas, resultados, tendências e gestão.

Para Kohama (1999, p. 174), a situação financeira de uma entidade pública é medida pelo quociente Ativo Financeiro dividido pelo Passivo Financeiro, o qual demonstra o quanto de disponibilidade financeira a entidade tem para saldar suas obrigações de curto prazo.

O problema em utilizar somente esse quociente para verificar a situação financeira de uma entidade está em não considerar alguns fatores importantes, como por exemplo, a capacidade de arrecadação de receitas próprias, as dívidas de longo prazo ou até mesmo a capacidade de gerar poupança.

Nesse sentido, Lopreato (2004, p. 5) informa que a condução das finanças públicas impõe o cumprimento de regras definidas pela legislação, quais sejam: limites para a dívida; normas de contratação de operações de crédito; parâmetros para determinados gastos; e critérios de eliminação do excesso de endividamento. Isso tudo para impedir que eventuais excessos gerem o descontrole das finanças públicas.

Para tratar do aspecto financeiro das entidades públicas, existem na literatura muitos indicadores de diferentes fontes. Dessa forma, foi adotado neste estudo os selecionados por Mello e Slomski (2006), que usaram análise fatorial para identificar os indicadores mais apropriados entre aqueles sugeridos pela sua literatura.

Os indicadores utilizados são os seguintes:

- Proporção de receitas que o Estado recebe do governo federal: relação entre as transferências federais e as receitas de fontes próprias (TF / RFP);

- Parcela do passivo total que é proveniente de capital de terceiros: relação entre ativo total menos o passivo total e a receita corrente líquida ((AT - PT) / $\mathrm{RCL})$;

* Parcela dos ativos que está comprometida com o endividamento: relação entre o passivo financeiro menos o ativo financeiro e o ativo total ((PF - AF) / AT); 
- Necessidade de recursos: relação entre o passivo financeiro menos o ativo financeiro e a receita corrente ((PF - AF) / RC);

- Participação da receita tributária: relação entre a receita tributária e a receita total (RTrib. / RT);

* Participação das receitas de transferência: relação entre as receitas de transferências e a receita total (RTransf. / RT);

* Participação do FPE: relação entre o fundo de participação do estado e a receita total (FPE / RT);

* Participação do ICMS: relação entre o imposto sobre circulação de mercadorias e serviços e a receita total (ICMS / RT);

- Receitas per capita: relação entre as receitas próprias e a população (RP / População);

* Financiamento das dívidas de custeio: relação entre a as dívidas de custeio e as despesas correntes (RT / DC);

- Cobertura corrente total: relação entre as receitas tributárias, menos as despesas correntes, e as receitas tributárias (RTrib. - DCor.) / RTrib.); e

* Cobertura corrente própria: relação entre as receitas tributárias com ICMS e as despesas correntes ((RTrib.+ ICMS) / DCor.).

Uma vez definidos os indicadores, cabe destacar o detalhamento da metodologia da pesquisa e dos procedimentos estatísticos empregados para auxiliar a conclusão sobre o tema.

\section{METODOLOGIA}

Para a concretização deste estudo, utilizou-se como técnica de trabalho a pesquisa empíco-analítica, que segundo Martins (2002, p. 34), “[...] são abordagens que apresentam em comum a utilização de técnicas de coleta, tratamento e análise de dados marcadamente quantitativos. Privilegiam estudos práticos. Suas propostas têm caráter técnico, restaurador e incrementalista."

Os dados utilizados para calcular os indicadores foram obtidos nos relatórios contábeis e financeiros do banco de dados da Secretaria do Tesouro Nacional e do Instituto Brasileiro de Geografia e Estatística (IBGE). Os indicadores foram calculados para todos os estados brasileiros, exceto o Distrito Federal, no período de 2000 a 2005. O Distrito Federal foi excluído porque a estrutura de seus gastos/ receitas é diferente da dos Estados analisados; o que poderia causar distorções nos resultados.

0 trabalho de pesquisa consiste em verificar quais indicadores podem, individualmente, explicar a situação financeira dos Estados, para então, através deles, propor uma função que melhor a represente. Para tal, foi utilizada uma técnica estatística de análise multivariada de dados, conhecida como "análise discriminante".

A análise discriminante, segundo Maroco (2003, p. 331) tem por objetivos:

a) identificação das variáveis que melhor discriminam entre dois ou mais grupos de indivíduos estruturalmente diferentes e mutuamente exclusivos;

b) a utilização destas variáveis para criar uma função discriminante que represente de forma parcimoniosa as diferenças entre os grupos; 
c) a utilização desta função discriminante para classificar à priori novos indivíduos nos grupos.

De acordo com Hair et al (2005, p. 209), “[...] a discriminação é conseguida estabelecendo-se os pesos da variável estatística para cada variável, para maximizar a variância entre grupos relativa à variância dentro dos grupos, e a função discriminante é determinada de uma equação".

A equação assume a seguinte forma:

$$
\mathrm{Z}_{\mathrm{jk}}=a+\mathrm{W}_{1} \mathrm{X}_{1 \mathrm{k}}+\mathrm{W}_{2} \mathrm{X}_{2 \mathrm{k}}+\ldots+\mathrm{W}_{\mathrm{n}} \mathrm{X}_{\mathrm{nk}}
$$

Em que:

$Z_{\mathrm{jk}}=$ escore $\mathbf{Z}$ discriminante da função discriminante $\mathrm{j}$ para o objeto $\mathrm{k}$.

$a=$ intercepto.

$\mathrm{W}_{1}=$ peso discriminante para a variável independente 1 .

$\mathbf{X}_{1 \mathrm{k}}=$ variável independente 1 para o objeto $\mathrm{k}$.

Essa função é conhecida como função discriminante linear de Fisher, onde após a dedução da primeira função discriminante, os pesos das funções seguintes são obtidos sobre a restrição adicional de que os escores das funções não estejam correlacionados (MAROCO, 2003, p. 334).

Entretanto, para que essa técnica possa ser empregada, algumas condições devem ser observadas, tais como: normalidade multivariada das variáveis independentes; homogeneidade das matrizes de variância e covariância; ausência de multicolinearidade e linearidade (HAIR et al, 2005, p. 220-221).

Segundo Tabachnick e Fidell (2001, p. 462), a normalidade multivariada significa que as variáveis independentes formam amostras aleatoriamente escolhidas da população e que a distribuição de seus valores se aproxima de uma distribuição normal. De acordo com Maroco (2003, p. 332), não existe nenhum teste para verificar esse pressuposto, mas de uma forma geral, aceita-se que cada uma das $p$ variáveis possui distribuição normal.

Com relação à condição de homogeneidade das matrizes de variância e covariância, Hair (2005, p. 220) explica que as matrizes de covariância desiguais podem afetar negativamente o processo de classificação. Se os tamanhos das amostras são pequenos e as matrizes são diferentes, a significância estatística do processo de estimação é afetada adversamente.

A multicolinearidade denota que duas ou mais variáveis independentes estão altamente correlacionadas, de modo que uma variável pode ser altamente explicada ou prevista pela outra variável (ou outras variáveis), acrescentando pouco ao poder explicativo do conjunto (HAIR et al, 2005, p. 221).

Por último, quanto à linearidade das variáveis, Hair et al (2005, p. 221) informa que é uma suposição implícita, pois as relações não lineares não são refletidas na função discriminante, a menos que transformações específicas de variáveis sejam executadas para representar efeitos não lineares.

Outro ponto importante da análise discriminante é a classificação dos grupos, ou a determinação do escore de corte, que segundo Hair (2005, p. 224) “[...] é o critério 
(escore) em relação ao qual o escore discriminante de cada objeto é comparado para determinar em qual grupo o objeto deve ser classificado".

O escore de corte também é chamado de $Z$ crítico, que dependendo dos tamanhos dos grupos, podem ser definidos, de acordo com Hair (2005, p. 224), como:

$\rightarrow \quad$ Escore de corte para dois grupos de mesmo tamanho $=Z_{C E}=\left(Z_{A}+Z_{B}\right) / 2$; em que:

$Z_{C E}=$ valor do escore de corte crítico para grupos de mesmo tamanho

$Z_{A}=$ centróide do grupo $A$

$Z_{B}=$ centróide do grupo $B$

$+\quad$ Escore de corte para diferentes tamanhos de grupos $=Z_{C U}=\left(N_{A} Z_{B}+N_{B} Z_{A}\right) /$ $\left(N_{A}+N_{B}\right)$; em que:

$Z_{C U}=$ valor de escore de corte crítico para grupos com tamanhos diferentes.

$N_{A}=$ número no grupo $A$.

$N_{B}=$ número no grupo $B$.

$Z_{A}=$ centróide para o grupo $A$.

$Z_{B}=$ centróide para o grupo $B$.

Quanto à parte prática, o primeiro passo foi aplicar as fórmulas e obter os resultados dos indicadores descritos na revisão bibliográfica, nos anos de 2000 a 2005, em todos os estados brasileiros, totalizando 156 observações para cada indicador, para então, fazer a padronização, classificando os resultados dos indicadores em uma escala de 1 a 20, considerando o valor mínimo, o máximo e o intervalo (STEVENSON, 2001).

A classificação dos indicadores acima descrita se fez necessária, pois, de acordo com Hair (2005, p. 221), as observações atípicas podem ter um impacto substancial na precisão da classificação de quaisquer resultados da análise multivariada de dados.

Depois disso, estabeleceram-se as variáveis independentes (Quadro 1); e para a variável dependente, considerou-se o indicador sugerido por Kohama (1999, p. 174), o qual estabelece a parcela das obrigações que as disponibilidades possam saldar (Ativo Financeiro dividido pelo Passivo Financeiro - AF/PF). Portanto, para os resultados do indicador $\mathrm{AF} / \mathrm{PF}$ acima de 1, considerou-se com disponibilidade financeira, identificada com o código 1 (um); e para os resultados abaixo de 1, considerou-se sem disponibilidade financeira, identificada com o código 0. 0 Quadro 1 mostra as variáveis independentes. 
Quadro 1 - Variáveis independentes

\begin{tabular}{|c|c|}
\hline Variáveis & Indicadores \\
\hline VAR 01 & (TF / RFP) \\
\hline VAR 02 & $((\mathrm{PF}-\mathrm{AF}) / \mathrm{AT})$ \\
\hline VAR 03 & $((\mathrm{PF}-\mathrm{AF}) / \mathrm{RC})$ \\
\hline VAR 04 & (RTrib. / RT) \\
\hline VAR 05 & (RTransf. / RT) \\
\hline VAR 06 & (FPE / RT) \\
\hline VAR 07 & (ICMS / RT) \\
\hline VAR 08 & (RP / População) \\
\hline VAR 09 & (RT / DC) \\
\hline VAR 10 & ((RTrib. - DCor.) / RTrib.) \\
\hline VAR 11 & ((RTrib.+ ICMS) - DCor.) \\
\hline VAR 12 & $((\mathrm{AT}-\mathrm{PT}) / \mathrm{RCL})$ \\
\hline
\end{tabular}

FONTE: Elaborado pelos autores.

Após o estabelecimento das variáveis, é necessário fazer a divisão da amostra. Segundo Hair (2005, p. 220), o procedimento mais popular envolve desenvolver a função discriminante em um grupo e então testá-la em um segundo grupo. Isso ocorre dividindo a amostra total de respondentes aleatoriamente em dois grupos, uma amostra de análise (usada para desenvolver a função discriminante); e uma amostra de teste (usada para testar a função). Esse método de validação da função é chamado de validação cruzada.

Dessa forma, foram divididas, aleatoriamente, as 156 observações de cada variável, com o indicativo para o programa de aproximadamente 60\%; e amostra de teste com aproximadamente $40 \%$.

Foi utilizado o software SPSS ${ }^{\circledR}$ - Statistical Package for Social Sciencies, versão 13.0, como ferramenta para execução dos testes estatísticos em um nível de significância de 0,05 .

\section{APRESENTAÇÃO E ANÁLISE DOS RESULTADOS}

Após a análise discriminante, o primeiro passo é avaliar as variáveis independentes, verificando as diferenças entre as médias.

Segundo Maroco (2003, p. 351) trata-se de testar a hipótese de que as médias dos grupos são iguais, e que entre as variáveis, pelo menos em um grupo as médias são diferentes; nesse caso, o objetivo é rejeitar a hipótese nula.

Dessa forma, as hipóteses testadas são as seguintes:

$+\quad \mathrm{H}_{0}=$ média dos dois grupos são iguais; e 
$+\quad \mathrm{H}_{1}=$ média dos dois grupos são diferentes.

0 resultado desse teste está na Tabela 1, na qual apenas as variáveis VAR 01 e VAR 10 não rejeitam a hipótese nula $\left(\mathrm{H}_{0}\right)$, pois o $p$-value (sig.) é maior do que o $\alpha$ (nível de significância) de 0,05 . Com isso, essas duas variáveis não passaram no pressuposto da igualdade das matrizes de variância e covariância, ou seja, não são significantes na diferenciação entre os grupos.

O segundo teste é a igualdade das matrizes de variância e covariância. Neste caso, o teste utilizado é o M de Box, que segundo Hair et al (2005, p. 207), é um teste estatístico utilizado para esse fim. Se o $p$-value (sig.) for maior do que o nível de significância, então a igualdade das matrizes encontra sustentação; se for menor, a suposição é violada. Logo, o objetivo é não rejeitar a hipótese que as matrizes são homogêneas.

Tabela 1 - Teste de Igualdade das Médias do Grup

\begin{tabular}{|c|c|c|c|c|c|} 
& $\begin{array}{c}\text { Wilks } \\
\text { Lambda }\end{array}$ & $\mathbf{F}$ & $\mathbf{d f 1}$ & $\mathbf{d f 2}$ & Sig. \\
\hline VAR 01 &, 991 & 0,445 & 1 & 51 &, 508 \\
\hline VAR 02 &, 595 & 34,712 & 1 & 51 &, 000 \\
\hline VAR 03 &, 538 & 43,876 & 1 & 51 &, 000 \\
\hline VAR 04 &, 816 & 11,496 & 1 & 51 &, 001 \\
\hline VAR 05 &, 850 & 9,034 & 1 & 51 &, 004 \\
\hline VAR 06 &, 833 & 10,257 & 1 & 51 &, 002 \\
\hline VAR 07 &, 831 & 10,367 & 1 & 51 &, 002 \\
\hline VAR 08 &, 678 & 24,274 & 1 & 51 &, 000 \\
\hline VAR 09 &, 881 & 6,883 & 1 & 51 &, 011 \\
\hline VAR 10 &, 952 & 2,595 & 1 & 51 &, 113 \\
\hline VAR 11 &, 863 & 8,124 & 1 & 51 &, 006 \\
\hline VAR 12 &, 530 & 45,146 & 1 & 51 &, 000 \\
\hline
\end{tabular}

FONTE: Elaborada pelos autores.

As hipóteses a serem testadas são as seguintes:

$+\mathrm{H}_{0}=$ matrizes homogêneas; $\mathrm{e}$

$+\mathrm{H}_{1}=$ matrizes não homogêneas.

Tabela 2 - Resultado do Teste de Box’s M

\begin{tabular}{l|l|r}
\hline Box`s M & Approx. & 7,505 \\
\hline F & df1 & 2,395 \\
\hline & df2 & 3 \\
\hline & Sig. & 830389,9 \\
\hline
\end{tabular}

Fonte: Elaborada pelos autores.

0 resultado desse teste está na Tabela 2 , na qual o $p$-value (sig.) de 0,067 é maior do que o $\alpha$ (nível de significância) de 0,05 . Com isso, não se rejeita a $\mathrm{H}_{0}$, passando no pressuposto da igualdade das matrizes. 
A Tabela 3 evidencia o valor do Eigenvalue ou "autovalor", que segundo Maroco (2003, p. 353), é uma medida relativa de quão diferente os grupos são na função discriminante. Nesse caso, o resultado é de apenas uma função, correspondendo a $100 \%$ da variância explicada em termos de diferenças entre grupos. Essa tabela também apresenta a correlação canônica ${ }^{1}$, que demonstra o nível de associação entre os escores discriminantes e os grupos, ou seja, 0,723 é quanto o modelo explica da variável dependente.

Tabela 3 - Aultovalor

\begin{tabular}{c|c|c|c|c}
\hline Função & Autovalor & $\begin{array}{c}\text { \% de } \\
\text { Variancia }\end{array}$ & $\begin{array}{c}\text { Cumulativo } \\
\text { \% }\end{array}$ & $\begin{array}{c}\text { Correlação } \\
\text { Canônica }\end{array}$ \\
\hline 1 & 1,093 & 100,0 & 100,0 &, 723 \\
\hline
\end{tabular}

Fonte: Elaborada pelos autores.

O próximo teste de hipótese é o de Lambda de Wilks, que segundo Maroco (2003, p. 344) serve para testar a significância das funções discriminantes e é calculado a partir do determinante da matriz da soma dos quadrados e produtos cruzados dentro dos grupos; e do determinante da matriz da soma dos quadrados e produtos cruzados total.

As hipóteses a serem testadas são as seguintes:

- $\quad \mathrm{H}_{0}=$ média populacional dos dois grupos são iguais; $\mathrm{e}$

$\rightarrow \quad \mathrm{H}_{1}=$ média populacional dos dois grupos são diferentes.

O objetivo do teste é não aceitar a $\mathrm{H}_{0}$, pois as médias devem ser significativamente diferentes para melhor discriminar os grupos. 0 resultado desse teste está na Tabela 4, na qual o $p$-value (sig.) é menor do que o $\alpha$ (nível de significância) de 0,05 . Com isso, não se aceita a $\mathrm{H}_{0}$, concluindo que a função discriminante é altamente significativa.

Tabela 4 - Wilks` Lambda

\begin{tabular}{c|c|c|c|c}
\hline $\begin{array}{c}\text { Teste da } \\
\text { Função(s) }\end{array}$ & $\begin{array}{c}\text { Wilks' } \\
\text { Lambda }\end{array}$ & Chi-square & df & Sig. \\
\hline 1 &, 478 & 36,929 & 2 &, 000 \\
\hline
\end{tabular}

Fonte: Elaborada pelos autores.

A Tabela 5 apresenta os coeficientes estruturais, os quais estão agrupados de acordo com a correlação dentro dos grupos, entre as variáveis discriminantes VAR 12 e VAR 8 e a função. As variáveis estão ordenadas pelo grau absoluto de correlação dentro da função.

1 - A correlação canônica é um caso especial da correlação entre dois grupos de variáveis analisados em conjunto. $\mathrm{Na}$ análise discriminante a correlação é entre um grupo de variáveis discretas que identificam os grupos e as variáveis discriminantes (MAROCO, 2003, p. 353). 
Tabela 5 - Estrutura da Matriz

Tabela 6 - Coeficientes da Função Discriminante Canônica

\begin{tabular}{c|c}
\hline \multirow{2}{*}{ Variáveis } & Função \\
\cline { 2 - 2 } & $\mathbf{1}$ \\
\hline VAR 08 &, 140 \\
\hline VAR 12 &, 314 \\
\hline Constante & $-3,841$ \\
\hline
\end{tabular}

Coeficientes não padronizados.

Fonte: Elaborada pelos autores.

a. Estas variáveis não são usadas na análise:

\begin{tabular}{|c|c|}
\hline \multirow{2}{*}{ Variáveis } & Função \\
\hline & 1 \\
\hline VAR 12 & 900 \\
\hline VAR $03^{\mathrm{a}}$ & ,894 \\
\hline VAR $02^{\mathrm{a}}$ & ,715 \\
\hline VAR 08 &, 660 \\
\hline VAR $09^{\mathrm{a}}$ &, 529 \\
\hline VAR $04^{\mathrm{a}}$ & ,524 \\
\hline VAR $07^{\mathrm{a}}$ & ,507 \\
\hline VAR $11^{\mathrm{a}}$ & ,487 \\
\hline VAR $06^{\mathrm{a}}$ &,- 460 \\
\hline VAR $10^{\mathrm{a}}$ & ,385 \\
\hline VAR $05^{\mathrm{a}}$ &,- 347 \\
\hline VAR $01^{\mathrm{a}}$ &,- 249 \\
\hline
\end{tabular}

Fonte: Elabora pelos autores

A próxima tabela (Tabela 6) apresenta as variáveis selecionadas para compor a função e seus respectivos coeficientes não padronizados.

Portanto, conforme os resultados apresentados na tabela acima, a função é descrita da seguinte forma:

$Z=-3,841+0,140$ VAR $08+0,314$ VAR 12

Em continuidade, na Tabela 7 tem-se o resultado da função de centróides de grupos, pelo qual é possível calcular o Z crítico, e como neste trabalho os grupos são de tamanhos distintos $(0=$ sem disponibilidade financeira, em 49 observações e $1=$ com disponibilidade financeira, em 44 observações, conforme a Tabela 9), o escore de corte é assim determinado:

$$
\begin{aligned}
& -Z_{C U}=\left(N_{A} Z_{B}+N_{B} Z_{A}\right) /\left(N_{A}+N_{B}\right) \\
& -Z_{C U}=(44(0,969)+49(-1,085)) /(44+49) \\
& -Z_{C U}=-0,11321
\end{aligned}
$$


Os tamanhos dos grupos usados no cálculo precedente são baseados no conjunto de dados empregados na amostra de análise e não incluem a amostra de validação.

0 procedimento para classificar a situação financeira dos Estados com o escore de corte ótimo é o seguinte: classifica-se um Estado como "sem disponibilidade financeira" se seu escore discriminante for menor do que -0,11321; e classifica-se um Estado como "com disponibilidade financeira" se seu escore discriminante for maior do que $-0,11321$. Veja-se

a Tabela 7.

Tabela 7 - Função de Centróides de Grupos

\begin{tabular}{c|c}
\hline \multirow{2}{*}{ Situação financeira } & Função \\
\cline { 2 - 2 } & $\mathbf{1}$ \\
\hline 0 &, 969 \\
\hline 1 & $-1,085$ \\
\hline
\end{tabular}

Fonte: Elaborada pelos autores.

A função de classificação de Fischer é apresentada na Tabela 8, na qual, as observações podem ser classificadas, considerando o grupo que apresentar maior escore. 0 procedimento classificatório consiste em introduzir os escores discriminantes e o valor das respectivas variáveis na função discriminante; o resultado maior indica a que grupo pertence aquele Estado (se é sem disponibilidade financeira ou com disponibilidade financeira).

Tabela 8 - Função de Classificação de Fisher's

\begin{tabular}{c|c|c}
\hline \multirow{2}{*}{} & \multicolumn{2}{|c}{ Situação Financeira } \\
\cline { 2 - 3 } & $\mathbf{0}$ & $\mathbf{1}$ \\
\hline VAR 08 &, 601 &, 313 \\
\hline VAR 12 & 1,578 &, 934 \\
\hline Constante & $-12,292$ & $-4,521$ \\
\hline
\end{tabular}

Fonte: Elaborada pelos autores.

Por fim, tem-se a validação dos resultados obtidos na análise discriminante. Neste processo, utilizaram-se duas formas: a validação interna; e a validação externa.

A validação interna, apresentada na Tabela 9, consiste em verificar a eficácia da classificação das observações originais e a validação cruzada. O resultado é que na classificação original, $81,1 \%$ das observações foram bem classificadas e na final, $77,4 \%$.

A validade externa, que confirma os resultados da validação interna, não é obtida juntamente com a análise discriminante, por isso, esse procedimento foi executado no software Excel $^{\circledR}$ a partir das observações da amostra teste. Foi executada a função discriminante para cada observação, levando em consideração o cálculo do escore discriminante ótimo. 0 resultado obtido foi que $75 \%$ das observações foram bem classificadas. Veja-se a Tabela 9. 
Tabela 9 - Classificação dos Resultados ${ }^{\text {ab }}$

\begin{tabular}{|c|c|c|c|c|c|}
\hline \multirow{2}{*}{\multicolumn{3}{|c|}{ Situação Financeira }} & \multicolumn{2}{|c|}{ Grupos } & \multirow[b]{2}{*}{ Total } \\
\hline & & & $\mathbf{0}$ & 1 & \\
\hline \multirow[t]{4}{*}{ Original } & \multirow[t]{2}{*}{ Count } & 0 & 35 & 14 & 49 \\
\hline & & 1 & 4 & 40 & 44 \\
\hline & \multirow[t]{2}{*}{$\%$} & 0 & 71,4 & 28,6 & 100,0 \\
\hline & & 1 & 8,0 & 92,0 & 100,0 \\
\hline \multirow[t]{4}{*}{ Validação Cruzada } & \multirow[t]{2}{*}{ Count } & 0 & 33 & 16 & 49 \\
\hline & & 1 & 5 & 39 & 44 \\
\hline & \multirow[t]{2}{*}{$\%$} & 0 & 67,9 & 32,1 & 100,0 \\
\hline & & 1 & 12 & 88 & 100,0 \\
\hline
\end{tabular}

a. $81,1 \%$ dos casos agrupados originalmente corretamente classificados.

b. $77,4 \%$ dos casos agrupados na validação cruzada corretamente classificados.

Fonte: Elaborada pelos autores.

Para melhor entendimento do processo de interpretação da situação financeira dos Estados, tem-se o seguinte exemplo hipotético: um determinado Estado, em 2005, teve como resultado da VAR 08 (RP / População), o valor 14²; e da VAR 12 ((AT - PT) / RCL), o valor $6^{2}$. Assim, pode-se calcular o resultado da função discriminante a seguir:

$$
Z=-3,841+0,140(14)+0,314(6)
$$

$$
Z=0,003
$$

Depois de encontrado o Z discriminante de 0,003, esse valor deve ser comparado com o escore de corte ótimo de -0,11321. Como 0,003 > -0,11321, classifica-se o Estado como "com disponibilidade financeira", considerando um nível de significância de $5 \%$.

\section{CONSIDERAÇÕES FINAIS}

Considerando os resultados gerais da análise discriminante, depois que os pressupostos foram atendidos, em que as variáveis selecionadas são significativamente discriminantes, e a própria função discriminante é altamente significante; pode-se concluir que as variáveis que representam a situação financeira dos estados brasileiros são:

* VAR 08 = fornece as receitas per capita, indicando a relação entre as receitas que possuem dependência com a geração e a população. É medida pelas receitas próprias divididas pela população; e

* VAR 12 = parcela do passivo total que é proveniente de capital de terceiros, indicando a relação entre o ativo total menos o passivo total e a receita corrente líquida ((AT - PT) / RCL); brasileiros é:

Assim sendo, a função que melhor representa a situação financeira dos estados

$$
Z=-3,841+0,140(\text { RP / População) + 0,314 ((AT - PT) / RCL) }
$$

\footnotetext{
2 - Esses valores são os padronizados, ou seja, são os valores originais classificados em uma escala de 1 a 20, conforme valores mínimos, máximos e o intervalo.
} 
As outras variáveis analisadas foram descartadas da análise discriminante porque não discriminaram tão bem quanto as escolhidas. A situação financeira não se explica por um único indicador, é necessária uma análise do conjunto de indicadores que, estatisticamente, a expliquem, diferentemente, de analisar um ou outro indicador sem saber seu poder de explicação.

Um fator limitador desta pesquisa é a quantidade de períodos analisados, pois foram considerados apenas seis anos; o que pode causar algumas distorções. Mas, enquanto não se tem um número maior de períodos para análise, por essas informações só estarem disponíveis a partir de 2000 (exigência da LRF), trabalha-se com essa limitação.

Como sugestão de novas pesquisas, pode-se verificar se esse resultado se confirma, aplicando-o nos municípios ou, futuramente, nos próprios estados, considerando-se um número maior de períodos.

\section{REFERÊNCIAS}

BRASIL. Ministério da Fazenda. Tesouro Nacional. Disponível em: <http://www.tesouro.fazenda.gov.br>.

. Ministério do planejamento, Orçamento e Gestão. IBGE - Instituto Brasileiro de Geografia e Estatística. Disponível em: <http://www.ibge.gov.br>.

CICA - CANADIAN INSTITUTE OFF CHARTERED ACCOUNTANTS. Indicators of government financial condition. Research report. Canada: 1997.

CARDOSO, Eloy S. Os vazios cofres dos estados e municípios. Revista Mercosul Magazine. 16/12/2003. Disponível em: <http://www.mercosulshop.com.br/conteudo/View_Article_ Stage_3.asp?Article_ID=602>. Acesso em: 10/04/2006.

DUNCAMBE, William; JUMP, Bernard; AMMAR, Salwa; WRIGHT, Ronald. Developing a financial condition indicator system for New York School Districts. Maio/2003. <Disponível em: www.albany.edu/edfin/cr03.wd.etal.fcis.schools.pdf>. Acesso em: 11/04/06.

HAIR, Joseph F.; ANDERSON, Rolph E.; TATHAM, Ronald L.; BLACK, William C. Análise multivariada de dados. Porto Alegre: Bookman, 2005. Tradução da $5^{a}$ edição americana por Adonai Schlup Sant 'Anna e Anselmo Chaves Neto.

KOHAMA, Heilio. Balanços públicos - teoria e prática. São Paulo: Atlas, 1999.

LOPREATO, Francisco Luiz. A situação financeira dos Estados e a reforma tributária. Texto para Discussão. IE/UNICAMP, Campinas, n. 115, mar. 2004.

MAROCO, João. Análise estatística: com a utilização do SPSS. Lisboa: Sílabo, 2003.

MARTINS, Gilberto de Andrade. Manual para elaboração de monografias e dissertações. 3 ed. São Paulo: Atlas, 2002.

MATIAS, Alberto Borges; CAMPELLO, Carlos A. G. B. Administração Financeira Municipal. São Paulo: Atlas, 2000.

MELLO, Gilmar Ribeiro de; SLOMSKI, Valmor. Verificando o Endividamento dos Estados Brasileiros: Uma Proposta Utilizando Análise Multivariada de Dados. Anais do $30^{\circ}$ EnANPAD, 2006.

OFFICE OF THE STA COMPTROLLER. Financial condition analysis. Local Government Management Guide. Nova York: 2002. 
STEVENSON, William J. Estatística aplicada à administração. São Paulo: Harbra, 2001. Traduzido por Alfredo Alves de Farias.

TABACHNICK, Barbara G.; FIDELL, Linda S. Using multivariate statistics. 4 ed. Boston: Allyn and Bacon, 2001.

\section{ENDEREÇO DOS AUTORES}

Universidade Estadual do Oeste do Paraná Centro de Ciências Sociais Aplicadas

Campus de Francisco Beltrão

R. Maringa, 1200 - Vila Nova

Francisco Beltrao, PR - Brasil

85600-000
Universidade de São Paulo

Faculdade de Economia, Administração e Contabilidade Departamento de Contabilidade e Atuária

R. Professor Luciano Gualberto, 908 - Cidade Universitária

Sao Paulo, SP - Brasil

05508900 